\title{
Search for the associated production of a Higgs boson and a top quark pair in multilepton (2 leptons, no hadronically-decaying $\tau$ lepton candidates and 4 leptons) final states with the ATLAS detector.
}

\author{
Dumitriu Ana Elena*i \\ IFIN-HH Bucharest, CPPM Marseille \\ E-mail: ana.elena.dumitriudcern.ch
}

\begin{abstract}
The Yukawa coupling of the Higgs boson to the top quark is a key parameter of the Standard Model. It can be constrained using the associated production process $p p \rightarrow t \bar{t} H+X$. A search for this process using final states with multiple leptons, primarily targeting the decays $H \rightarrow W W^{*}$ and $H \rightarrow \tau \tau$, has been performed using the data set recorded by the ATLAS detector in 2015 and 2016 at a center of mass energy $\sqrt{s}=13 \mathrm{TeV}$. The analysis presented here includes two of the four final states distinguished by the number and flavor of leptons: two same-charge light leptons (e or $\mu$ ) and no hadronically-decaying $\tau$ lepton candidates ( $\left.2 \ell 0 \tau_{\text {had }}\right)$ and four light leptons ( $4 \ell$ ), the remaining channels not covered being two same-charge light leptons and one hadronicallydecaying $\tau$ lepton candidate $\left(2 \ell 1 \tau_{\text {had }}\right)$ and three light leptons $(3 \ell)$. The different background sources are also presented for each channel considered. The latest best-fit value for the ratio of observed versus Standard Model cross sections is then given as well as an upper limit on this parameter.
\end{abstract}

The European Physical Society Conference on High Energy Physics

5-12 July, 2017

Venice

${ }^{*}$ Speaker.

On behalf of the ATLAS Collaboration 


\section{Introduction}

The Yukawa coupling of the Higgs boson to the top quark is a key parameter of the Standard Model (SM) opening potential window to signs of new physics that can modify the $t \bar{t} H$ production cross section relative to the SM expectation. This parameter can be determined from the ratio of the top quark mass and Higgs field vacuum expectation value, from the cross section of $g g \rightarrow H$ production through a top quark loop, or from the cross section of the tree-level process $g g / q q \rightarrow$ $t \bar{t} H$.

In this analysis are presented the results of a search for $t \bar{t} H$ production using $13.2 \mathrm{fb}^{-1}$ of data collected with the ATLAS detector [1] at $\sqrt{s}=13 \mathrm{TeV}$ during 2015 and 2016. The search is based on four final states distinguished by the number and flavor of leptons: two same-charge light leptons (e or $\mu$ ) and no hadronically-decaying $\tau$ lepton candidates $\left(2 \ell 0 \tau_{\text {had }}\right)$; two same-charge light leptons and one hadronically-decaying $\tau$ lepton candidate $\left(2 \ell 1 \tau_{\text {had }}\right)$; three light leptons ( $\left.3 \ell\right)$;

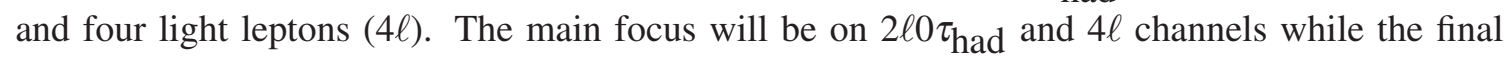
result are presented for all channels combined. Backgrounds are estimated with a combination of simulation and data-driven techniques, while the global fit to the yields in all final states is used to extract the best estimate for the $t \bar{t} H$ production rate.

\section{Object and Event Preselection}

Events are required to be selected by single electron or muon triggers (94 to $99 \%$ efficient). The primary vertex in an event is chosen as the vertex with the highest $\Sigma p_{\mathrm{T}}^{2}$ of associated tracks.

Electron candidates are reconstructed from energy clusters in the electromagnetic calorimeter that are associated with charged particle tracks reconstructed in the inner detector [2]. Only candidates with $p_{\mathrm{T}}>10 \mathrm{GeV}$ and $\left|\eta_{\text {cluster }}\right|<2.47$ are considered. Isolation variables are used to reduce the background from non-prompt electrons produced in hadron decays.

Muon candidates are reconstructed by combining inner detector tracks with track segments or full tracks in the muon spectrometer [3]. Candidates are required to satisfy $p_{\mathrm{T}}>10 \mathrm{GeV}$ and $|\eta|<$ 2.5 .

Jets are reconstructed from calibrated topological clusters built from energy deposits in the calorimeters [4], using the anti- $k_{t}$ algorithm with a radius parameter $R=0.4$, and then only jets satisfying $p_{\mathrm{T}}>25 \mathrm{GeV}$ and $|\eta|<2.5$ are considered. Jets containing $b$-hadrons are identified ( $b$ tagged) via a multivariate discriminant, the working point used for this search corresponds to an average efficiency of $70 \%$ for $b$-jets with $p_{\mathrm{T}}>20 \mathrm{GeV}$ and $|\eta|<2.5$ in simulated $t \bar{t}$ events.

Missing transverse momentum ( $E_{\mathrm{T}}^{\text {miss }}$ ) [5] is used to define certain additional selections for validation of background modeling. The missing transverse momentum vector is defined as minus the vector sum of the transverse momenta of all reconstructed and calibrated physics objects and remaining unclustered energy.

\section{Channel Selection}

For the $2 \ell 0 \tau_{\text {had }}$ channel, the two light leptons with transverse momentum $p_{\mathrm{T}}>25 \mathrm{GeV}$ and with same charge are selected and sorted into three subcategories based on the flavor of the leptons 
$(e e, e \mu, \mu \mu)$. Events must have $\geq 5$ jets, of which $\geq 1$ must be $b$-tagged and no $\tau_{\text {had }}$ candidates.

In the case $4 \ell$ channel, the sum of the charges of the leptons in these events must be zero. All leptons must pass the gradient isolation selection. To reject $t \bar{t} Z$ and $Z Z$ background and to remove leptons from quarkonium decays, all same-flavor $\ell^{+} \ell^{-}$pairs in the event must satisfy $m_{\ell^{+} \ell^{-}}-91.2$ $\mathrm{GeV} \mid>10 \mathrm{GeV}$ respectively $m_{\ell^{+} \ell^{-}}>12 \mathrm{GeV}$. The four-lepton invariant mass must satisfy 100 $\mathrm{GeV}<m_{4 \ell}<350 \mathrm{GeV}$ and $\left|m_{4 \ell}-125 \mathrm{GeV}\right|>5 \mathrm{GeV}$ to reduce contamination from $Z \rightarrow 4 \ell$ at low mass and $t \bar{t} Z$ at high mass as well as to reduce contamination from other Higgs boson production processes. There must be at least two jets in the event, of which at least one must be $b$-tagged.

\section{Backgrounds}

Backgrounds in the signal regions are categorized as prompt leptons (all the selected leptons are produced in decays of electroweak bosons or $\tau$ ) and non-prompt (charge misreconstruction or fake) leptons that arise from hadron decays or photon conversions, other interactions in detector material, or improper reconstruction of other particle species.

Backgrounds with Prompt Leptons are estimated using Monte Carlo (MC) simulation. In all final states, $t \bar{t} V$ production is the largest background with prompt leptons, followed by diboson production and rarer processes. Systematic uncertainties on the acceptance for the $t \bar{t} V$ backgrounds are derived using MC event simulation. The largest backgrounds with prompt leptons are studied in validation regions (VR) that are disjoint from the signal regions and enhance specific processes. These permit checks of the overall normalization and jet multiplicity dependence of specific backgrounds.

Charge misreconstruction affects the $2 \ell 0 \tau_{\text {had }}$ categories with electrons while for $4 \ell$ channels is negligible: an event with an opposite-charge light lepton pair is made to appear as a same-charge pair. The rates for charge misreconstruction are determined from data using dielectron events with $m(e e)$ consistent with the $Z$ mass. Charge misreconstruction for muons is negligible in this analysis.

Non-prompt leptons in the $2 \ell 0 \tau_{\text {had }}$ channels are estimated by extrapolating from control regions (CR) dominated by such backgrounds into the signal region. The ratio of non-prompt leptons passing tight and anti-tight (pass the loose but not tight) selections is measured from data, and gives transfer factors for non-prompt electrons and muons, then applied to events that pass signal region (SR) selections except for having one tight and one anti-tight lepton, giving an estimate of the yield of events with non-prompt leptons in the signal region. The systematic uncertainties on the transfer factors arise from the charge misreconstruction estimate, the extrapolation from the low jet multiplicity region to the signal regions, and statistical uncertainties in the data yields in the control regions.

To estimate the non-prompt leptons in the $4 \ell$ channels, corrected MC by non-prompt scale factors (NPSFs) determined from data-MC comparison in trilepton control regions, procedure separately done for electrons and muons and for processes with isolated production of high- $p_{\mathrm{T}} b$-quarks ( $t \bar{t}$-like) and processes with $g \rightarrow b \bar{b}$-dominated production of low- $p_{\mathrm{T}} b$-quarks ( $Z+$ jets-like), is used. NPSFs values range from $0.77 \pm 0.12$ (muon $t \bar{t}$-like) to $1.35 \pm 0.17$ (electron $Z+$ jets-like), where the quoted uncertainties are statistical only. Systematic uncertainties are estimated from variations in the event selection (20\%) and in the MC generators used (50\%). 


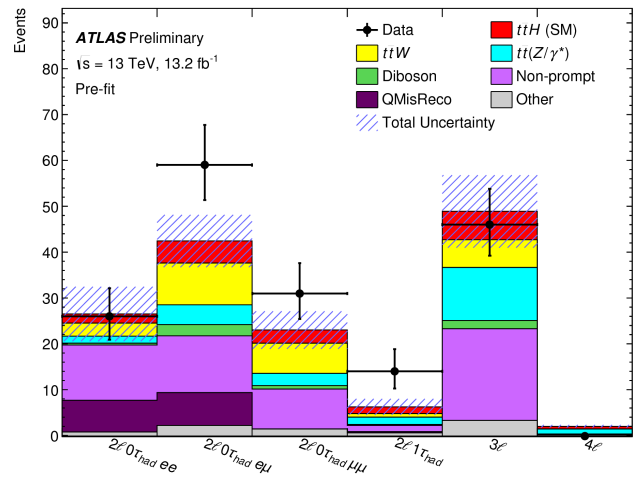

Figure 1: Summary of data vs estimated prefit events in the different channels [6].

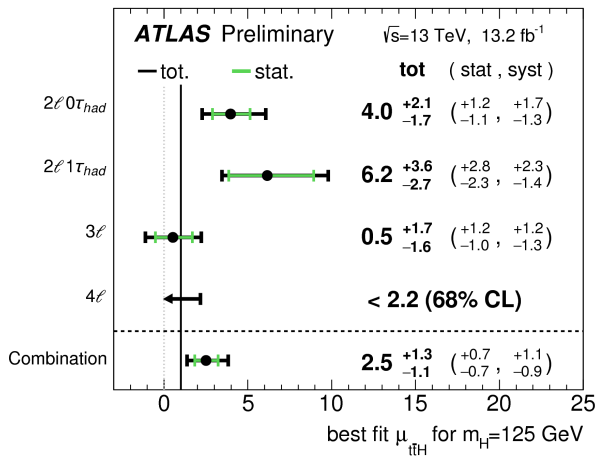

Figure 2: Measured signal strength $\mu$ in data for individual channels and their combination [6].

\section{Results and Conclusion}

The best-fit value of the $t \bar{t} H$ signal strength is obtained using a maximum likelihood fit to the data yields of the six categories shown in Figure 1. The best-fit value of $\mu_{t \bar{t} H}$ for four different final states is shown in Figure 2 and the combined best-fit $\mu_{t \bar{t} H}$ value is $2.5 \pm 0.7$ (stat) ${ }_{-0.9}^{+1.1}$ (syst), which is consistent with the Standard Model expectation. At 95\% confidence level, $\mu_{t \bar{t} H}$ is found to be less than 4.9 ( 2.3 expected).

\section{References}

[1] ATLAS Collaboration, The ATLAS Experiment at the CERN Large Hadron Collider, , JINST 3 (2008) S08003.

[2] ATLAS Collaboration, Electron efficiency measurements with the ATLAS detector using the 2015 LHC proton-proton collision data, ATLAS-CONF-2016-024, url: https://cds.cern.ch/record/2157687.

[3] ATLAS Collaboration, Measurement of the muon reconstruction performance of the ATLAS detector using 2011 and 2012 LHC proton-proton collision data, Eur. Phys. J. C 74 (2014) 3130, arXiv: 1407.3935 [hep-ex].

[4] ATLAS Collaboration, Jet Calibration and Systematic Uncertainties for Jets Reconstructed in the ATLAS Detector at $\sqrt{s}=13 \mathrm{TeV}$ ), ATL-PHYS-PUB-2015-015, url: https://cds.cern.ch/record/2037613.

[5] ATLAS Collaboration, Performance of missing transverse momentum reconstruction for the ATLAS detector in the first proton-proton collisions at at $\sqrt{s}=13 \mathrm{TeV}$, ATL-PHYS-PUB-2015-027, url: https://cds.cern.ch/record/2037904.

[6] ATLAS Collaboration. Search for the Associated Production of a Higgs Boson and a Top Quark Pair in Multilepton Final States with the ATLAS Detector. ATLAS-CONF-2016-058, 2016. 